\title{
Features Design of Organic Rankine Cycle
}

\author{
Denis I. Karabarin* and Sergei A. Mihailenko \\ Siberian Federal University \\ 79 Svobodny, Krasnoyarsk, 660041, Russia
}

Received 05.10.2018, received in revised form 10.03.2019, accepted 20.07.2019

This article discusses the design features of plants operating on the principle of organic Rankine cycle. Firstly, the choice of organic Rankine cycle as the most effective technology for utilization of low-potential heat by comparison and analysis with other technologies is justified. Secondly, the technique of selection and calculation of the installations working on the principle of an organic Rankine cycle, taking into account features of a choice of a working body, type of the expander, and also a configuration is offered. Third, an example of the calculation of the prototype of such a $4 \mathrm{~kW}$ unit operating on the thermal energy of hot water from the boiler, the simulation of which is performed in the program SmoWeb, the results of which it was designed. Fourth, the design for this technology takes.

Keywords: organic Rankine cycle, utilization of low-grade heat, energy efficiency, organic coolant, expander.

Citation: Karabarin D.I., Mihailenko S.A. Features design of organic Rankine cycle, J. Sib. Fed. Univ. Eng. technol., 2019, 12(6), 733-745. DOI: 10.17516/1999-494X-0173.

\section{Особенности проектирования установок органического цикла Ренкина}

Д.И. Карабарин, С.А. Михайленко Сибирский федеральный университет Россия, 660041, Красноярск, пр. Свободный, 79

В этой статье рассмотрены особенности проектирования установок, работающих по принципу органического иикла Ренкина. Во-первых, обоснован выбор органического иикла Ренкина в качестве наиболее эффективной технологии для утилизации низкопотенциального тепла путем сравнения и анализа с другими технологиями. Во-вторых, предложена методика подбора и расчета установок, работающих по принщипу органического иикла Ренкина, с учетом особенностей выбора рабочего тела, типа расширителя, а также конфигурации. B-третьих, представлен пример расчета прототипа такой установки на 4 кBт, работающей

(C) Siberian Federal University. All rights reserved

This work is licensed under a Creative Commons Attribution-NonCommercial 4.0 International License (CC BY-NC 4.0)

* Corresponding author E-mail address: 18app@mail.ru 
на тепловой энергии горячей воды от котла, моделирование которого выполнено в программе SmoWeb, по результатам которого он был спроектирован. В-четвертых, в проектировании для данной технологии учтены особенности эксплуатации, пуска и ремонта.

Ключевые слова: органический иикл Ренкина, утилизаиия низкопотенциального тепла, энергоэффективность, органический теплоноситель, расширитель.

\section{Введение}

В связи с постоянным ростом выработки электрической и тепловой энергии, а также во исполнение Постановления Правительства РФ от 15 апреля 2014 г. № 321 “Об утверждении государственной программы Российской Федерации “Энергоэффективность и развитие энергетики” остро встает вопрос рационального использования любой энергии. В настоящее время актуальны вопросы автономного электроснабжения потребителей в районах децентрализованной энергетики и эффективная утилизация низкопотенциального сбросного тепла от производственных технологий в промышленности и энергетике. Принято считать, что источник энергии является низкопотенциальным, если его температура не превышает $230{ }^{\circ} \mathrm{C}$. Выбор рационального способа утилизации тепла позволил бы сократить издержки на собственные нужды в энергетике, машиностроении, металлургии, многих технологических процессах и т.д.

Как рассмотрено в [1-4], самой эффективной из существующих технологией использования низкопотенциальной теплоты признан органический цикл Ренкина (ОЦР) (рис. 1).

Источниками тепла для ОЦР могут быть:

- тепло от сжигания природного газа, пропана, доменного газа, пиролизного газа, свалочного газа, сланцевого газа, попутного (факельного) газа, биомассы;

- отходящее тепло от геотермальной энергии, отработанной горячей воды различных производств, пара паровых котлов или турбин, тепла выхлопа газовых турбин, отходящее тепло котельных установок;

- тепло солнечных термальных станций.
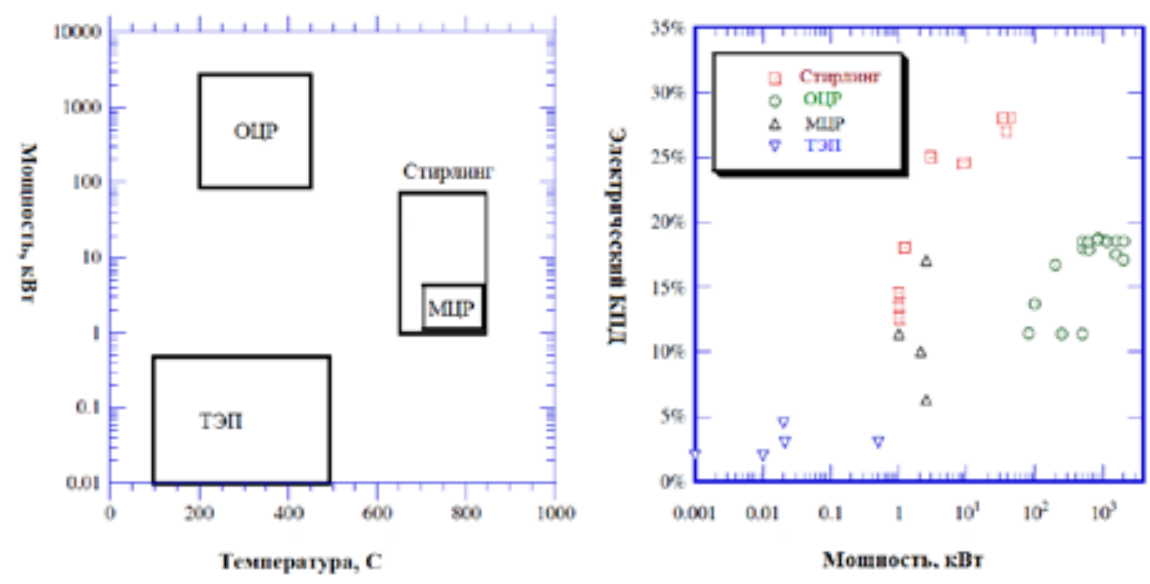

Рис. 1. Анализ технологий для использования низкопотенциального тепла [1]

Fig. 1. Analysis of technologies for using low potential heat [1]

$$
-734-
$$


Таблица 1. Основные производители и область внедрения установок ОЦР [5-12]

Table 1. The main manufacturers and the implementation area of ORC installations [5-12]

\begin{tabular}{|c|c|c|c|c|c|}
\hline Производитель & Область внедрения & $\begin{array}{c}\text { Температура } \\
\text { источника тепла }\end{array}$ & $\begin{array}{c}\text { Мощность, } \\
\text { кВт }\end{array}$ & $\begin{array}{l}\text { Рабочее } \\
\text { тело }\end{array}$ & Тип расширителя \\
\hline ORMAT, США & $\begin{array}{l}\text { Геотермальная, } \\
\text { утилизация, } \\
\text { солнечная }\end{array}$ & $150-300$ & $200-70,000$ & $\begin{array}{l}\text { Н-пентан и } \\
\text { другие }\end{array}$ & $\begin{array}{l}\text { Двухступенчатая } \\
\text { осевая турбина }\end{array}$ \\
\hline Turboden, Italy & $\begin{array}{l}\text { Биогазовая ТЭЦ, } \\
\text { утилизация, } \\
\text { геотермальная }\end{array}$ & $100-300$ & $200-2000$ & $\begin{array}{l}\text { OMTS, } \\
\text { Solkatherm }\end{array}$ & $\begin{array}{l}\text { Двухступенчатая } \\
\text { осевая турбина }\end{array}$ \\
\hline $\begin{array}{l}\text { Adoratec/ } \\
\text { Maxxtec, } \\
\text { Germany }\end{array}$ & Биогазовая ТЭЦ & 300 & $315-1600$ & OMTS & \\
\hline Opcon, Sweden & Утилизация & $<120$ & $350-800$ & Аммоний & Турбина Lysholm \\
\hline GMK, Germany & $\begin{array}{l}\text { Биогазовая ТЭЦ, } \\
\text { утилизация, } \\
\text { геотермальная }\end{array}$ & $120-350$ & $50-5000$ & & $\begin{array}{l}\text { Многоступенчатая } \\
\text { осевая турбина }\end{array}$ \\
\hline $\begin{array}{l}\text { Bosch KWK, } \\
\text { Germany }\end{array}$ & Утилизация & $120-150$ & $65-325$ & R245fa & \\
\hline $\begin{array}{l}\text { Turboden } \\
\text { PureCycle, US }\end{array}$ & $\begin{array}{l}\text { Утилизация, } \\
\text { геотермальная }\end{array}$ & $91-149$ & 280 & R245fa & $\begin{array}{l}\text { Радиальная } \\
\text { турбина }\end{array}$ \\
\hline GE CleanCycle & Утилизация & $>121$ & 125 & R245fa & $\begin{array}{l}\text { Одноступенчатая } \\
\text { радиальная } \\
\text { турбина }\end{array}$ \\
\hline Cryostar, France & $\begin{array}{l}\text { Утилизация, } \\
\text { геотермальная }\end{array}$ & $100-400$ & $\mathrm{n} / \mathrm{a}$ & $\begin{array}{l}\text { R245fa, } \\
\text { R134a }\end{array}$ & $\begin{array}{l}\text { Радиальная } \\
\text { турбина }\end{array}$ \\
\hline $\begin{array}{l}\text { Tri-o-gen, } \\
\text { Netherlands }\end{array}$ & Утилизация & $>350$ & 160 & Толуол & $\begin{array}{l}\text { Радиальная } \\
\text { турбина }\end{array}$ \\
\hline $\begin{array}{l}\text { Electratherm, } \\
\text { US }\end{array}$ & $\begin{array}{l}\text { Утилизация, } \\
\text { солнечная }\end{array}$ & $>93$ & 50 & R245fa & $\begin{array}{l}\text { Спиральный } \\
\text { рсширитель }\end{array}$ \\
\hline
\end{tabular}

Производство установок, работающих на основании ОЦР, начинается с 1980-х гг. Наиболее значимые производители и типы их установок представлены в табл. 1.

Как видно из данных табл. 1, для разных источников тепла и мощности различаются типы расширителей и рабочих сред. Наша статья и посвящена особенностям выбора рабочего тела и расширителя для проектирования установок, работающих на органическом цикле Ренкина.

\section{Выбор рабочего тела}

Рабочая жидкость очень важна для определения термодинамических свойств, экономической и технической составляющей для каждого цикла. Выбор подходящей рабочей жидкости должен учитывать термодинамическую составляющую, совместимость, стоимость и экологическую безопасность [13]. Интересное исследование по выбору жидкости представлено авторами [14]. Исследование рабочих жидкостей для ОЦР дано авторами [15], а в [16] изложено сравнение термофизических свойств различных рабочих жидкостей. В [17] было показано, что смеси рабочих тел улучшают характеристики цикла. В [18] обсуждаются преимущества смесей рабочих тел над чистыми рабочими телами с точки зрения безопасности и объемной эффектив-

$$
-735-
$$


ности. Автор [19] представил список потенциальных рабочих тел, подходящих для различных вариантов применения технологии ОЦР, сравнивая выбор рабочих тел в зависимости от объекта применения, температуры испарения и конденсации. Дальнейшие анализы по выбору жидкости были выполнены авторами [20]. Результаты анализа рабочих тел отражены в табл. 2.

Таблица 2. Анализируемые рабочие жидкости для разных систем

Table 2. Analyzed working fluids for different systems

\begin{tabular}{|c|c|c|c|c|}
\hline $\begin{array}{l}\text { Источник тепла/ } \\
\text { применение } \\
\text { технологии }\end{array}$ & Тконд. & Тисп. & Исследуемые тела & $\begin{array}{c}\text { Рекомендуемые } \\
\text { тела }\end{array}$ \\
\hline Утилизация тепла & $30-50$ & 120 & R11, R113, R142b, R114 & R113, R142b \\
\hline Неизв. & $35-60$ & $80-110$ & Неизв. & $\begin{array}{l}\text { RC123, R124, } \\
\text { R142b }\end{array}$ \\
\hline Утилизация тепла & 30 & $150-200$ & $\begin{array}{l}\text { RC123, HFE 7100, benzene, Toluene, } \\
\text { p-xylene }\end{array}$ & $\begin{array}{l}\text { Benzene, Toluene, } \\
\text { RC123 }\end{array}$ \\
\hline $\begin{array}{l}\text { Холодильная } \\
\text { промышленность }\end{array}$ & 55 & $60-150$ & $\begin{array}{l}\text { H2O, RC123, R142b, изопентан, R245ca, } \\
\text { R245fa, бутан, } \\
\text { изобутан, R152a }\end{array}$ & $\begin{array}{l}\text { H2O, R245ca, } \\
\text { R142b, Изобутан }\end{array}$ \\
\hline Когенерация & 90 & $250-350$ & $\begin{array}{l}\text { Бутил-бензен, пропил-бензен, этил- } \\
\text { бензен, } \\
\text { тоулен, OMTS, аммиак, R123, PF5050 }\end{array}$ & Бутил-бензен \\
\hline Не указано & $40-60$ & $220-350$ & HMDSO, OMTS, HMDSO/OMTS & HMDSO/OMTS \\
\hline Геотермальаная & 30 & 100 & $\begin{array}{l}\text { Алканы, фтор-алканы, эфиры, } \\
\text { фтороэфиры }\end{array}$ & $\begin{array}{l}\text { RE133, R245, } \\
\text { R600, R245fa, } \\
\text { R142b, R245ca, } \\
\text { R601 }\end{array}$ \\
\hline Геотермальаная & 25 & $80-115$ & $\begin{array}{l}\text { Пропилен, R227ea, RC318, R236fa, R142b, } \\
\text { изобутан, R245fa }\end{array}$ & $\begin{array}{l}\text { Пропилен, R227ea, } \\
\text { R245fa, R142b }\end{array}$ \\
\hline Утилизация тепла & 25 & $100-210$ & R113, R123, R245fa, R142b, изобутан & R113, R142b \\
\hline $\begin{array}{l}\text { Солнечная } \\
\text { энергия }\end{array}$ & 35 & $60-100$ & Хладоны & R152a, R600, R290 \\
\hline $\begin{array}{l}\text { Солнечная } \\
\text { энергия }\end{array}$ & 45 & $120-230$ & Н2О, н-пентан & н-пентан \\
\hline Утилизация тепла & 25 & 145 & $\begin{array}{l}\text { H2O, NH4, бутан, изобутан, R11, RC123, } \\
\text { R141B, R142b }\end{array}$ & бутан, R142b \\
\hline Утилизация тепла & 50 & $80-220$ & R600a, R245fa, RC123, R113 & R113, RC123 \\
\hline Когенерация & 50 & 170 & $\begin{array}{l}\text { R365mfc, гептан, пентан, R12, R141b, } \\
\text { этанол }\end{array}$ & этанол \\
\hline $\begin{array}{l}\text { Холодильная } \\
\text { промышленность }\end{array}$ & 76 & Неизв. & $\begin{array}{l}\text { R124 R134a, R245fa, R600, R600a, } \\
\text { R1234yf }\end{array}$ & R134a \\
\hline $\begin{array}{l}\text { Холодильная } \\
\text { промышленность }\end{array}$ & 95 & Неизв. & R125, R143a, R218 & R134a \\
\hline Геотермальаная & 30 & 150 & $\begin{array}{l}\text { R1225yeZ, R1234yf, R1234zeE, R1234zeZ, } \\
\text { R1234zf, R1225yeE, R1225zc, R1234yeE }\end{array}$ & R1234yf, R1225yeE \\
\hline Утилизация тепла & $20-35$ & 150 & $\begin{array}{l}\text { R245fa, R245fa/R152a, R245fa/R600a, } \\
\text { R113/R245fa, R601a/R600a, R236ea }\end{array}$ & Неизв. \\
\hline $\begin{array}{l}\text { Холодильная } \\
\text { промышленность }\end{array}$ & 35 & $96-221$ & R134, R11, бензен & бензен \\
\hline
\end{tabular}


Продолжение табл. 2

Continued table. 2

\begin{tabular}{|l|c|c|l|l|}
\hline Утилизация тепла & n/a & 120 & $\begin{array}{l}\text { R290, R600a, R601, R134a, R227ea, } \\
\text { R245fa, } \\
\text { R600a/R601, R290/R600a, R134a/R245fa }\end{array}$ & R600a/R601 \\
\hline Утилизация тепла & $27-87$ & 327 & $\begin{array}{l}\text { R245fa, R245ca, R236ea, R141b, R114, } \\
\text { RC123, } \\
\text { R113, R11, бутан }\end{array}$ & $\begin{array}{l}\text { R11, R141b, R113, } \\
\text { RC123, } \\
\text { R245fa, R245ca }\end{array}$ \\
\hline Утилизация тепла & n/a & 277 & R12, RC123, R134a, R717 & RC123 \\
\hline $\begin{array}{l}\text { Солнечная } \\
\text { энергия }\end{array}$ & 30 & 150 & n-pentane, SES36,R142b, R245fa, R134a & $\begin{array}{l}\text { R245fa, R142b, } \\
\text { R134a }\end{array}$ \\
\hline
\end{tabular}

Как видим из данных табл. 2, тип химического соединения рабочей жидкости зависит от температур испарения и конденсации. Для большинства вариантов, утилизирующих теплоту от производственных процессов при температуре источника тепла $80-150{ }^{\circ} \mathrm{C}$, рекомендуется использовать фреон R142b (C2F2CIH3), малоопасный и малотоксичный хладон, применяемый в качестве холодильного агента как для бытовых, так и для промышленных низкотемпературных установок.

\section{Выбор типа расширителя}

Выбор и определение размеров расширителя является одним из важнейших факторов, которые оказывают сильное влияние на стоимость и эффективность системы ОЦР. Расширители для системы ОЦР можно разделить на скоростные расширители (турбины) и расширители на основе объема (объемные расширители). Расширители на основе объема имеют специфическое встроенное объемное соотношение и могут быть классифицированы как лопастные расширители, поршневые расширители, винтовые расширители и спиральные расширители. Турбины для системы ОЦР могут быть радиальными или осевыми турбинами в зависимости от размера системы, массового расхода и отношения давления. Осевые турбины предпочтительны для большого массового расхода и низкой разности давления, в то время как радиальные турбины подходят для низкого массового расхода и высокой разности давлений. Однако турбины не подходят для небольших систем ОЦР (менее 50 кВт) в основном из-за высокой скорости вращения и высокой стоимости $[21,22]$. Как правило, низкопотенциальная рекуперация тепла с использованием ОЦР имеет выходную мощность 1-50 кВт [23-26], поэтому предпочтительны объемные расширители.

Объемные расширители характеризуются меньшими расходами, более высокими коэффициентами давления и значительно меньшими скоростями вращения по сравнению с расширителями скоростного типа. Они могут работать с двухфазной рабочей жидкостью, которая может появляется в конце процесса расширения в докритическом органическом цикле Ренкина. В объемных расширителях падение давления вызвано увеличением площади камеры расширения вдоль длины потока. Соотношение между камерами расширения в начале и в конце процесса расширения известно как встроенное соотношение объема расширителя. Фиксированное встроенное соотношение объема расширителя вызывает увеличение и уменьшение потерь. 
Изоэнтропическая эффективность расширителя будет ухудшаться, если удельное объемное отношение системы меньше, чем объемное соотношение расширителя. Встроенное соотношение объема объемных расширителей находится в диапазоне 2-6. За последние десять лет были проведены всесторонние исследования для оценки эффективности объемного расширителя для небольших установок по утилизации отходов. Большинство объемных расширителей, которые были изучены и проверены в этих исследованиях, были с модифицированной формой компрессора, реструктурированы для работы в обратном направлении в качестве расширителя. Объемные расширители можно разделить на лопастной расширитель, винтовой расширитель, поршневой расширитель и спиральный расширитель, как показано на рис. 2.

За несколько последних десятилетий компрессоры стали широко использоваться в кондитерской и холодильной промышленности. Компрессорная технология стала зрелой и коммерциализированной благодаря постоянным инновациям и совершенствованию технологий. В настоящее время наибольшая часть установок, утилизирующих низкопотенциальное и отработанное тепло, имеет расширитель, который является модифицированной формой компрессора. Рынок объемных расширителей еще не созрел, и доступно лишь несколько коммерческих объемных расширителей, в основном спиральные и винтовые. Существует ряд факторов, которые влияют на выбор расширительной машины для отработанного и низкопотенциального восстановления тепла. Эти факторы включают в себя эффективность, скорость вращения, смазку, потери на утечки, потери на трение, диапазон номинальной мощности, номинальную производительность, сложность, надежность, стоимость и доступность.

На основе экспериментальных данных из доступных публикаций известна сравнительная оценка для выбора и ранжирования объемных расширителей на основе их общей производи-
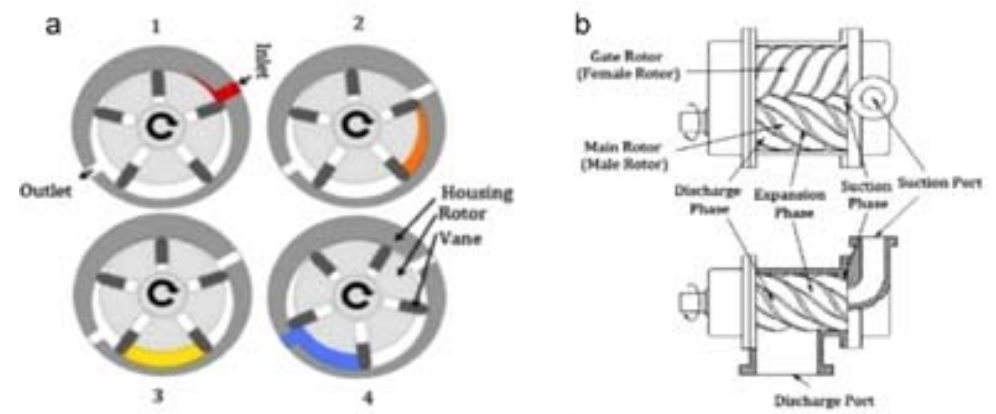

C

d
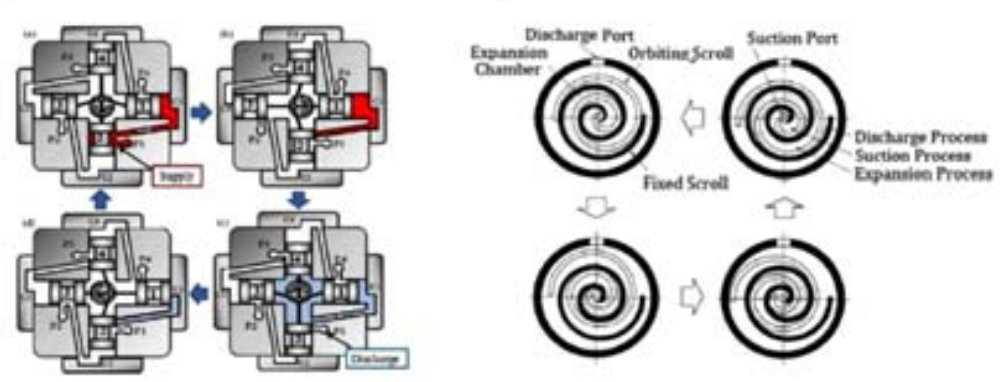

Рис. 2. Типы объемных расширителей: $\mathrm{a}$ - лопастной, $\mathrm{b}$ - винтовой, $\mathrm{c}-$ поршневой, $\mathrm{d}$ - спиральный

Fig. 2. Types of volumetric expanders: $a-$ blade: $b-$ screw: $c-$ piston: $d-$ spiral

$$
-738-
$$


тельности, стоимости, технических ограничений и статуса коммерциализации. Это исследование даст представление о выборе подходящего объемного расширителя для отработанного тепла и низкопотенциальной утилизации тепла, основанной на их характеристиках и экономике.

Лопастные расширители имеют простую конструкцию, низкую стоимость, высокий крутящий момент, высокую объемную эффективность и могут работать при высокой температуре и давлении. Они имеют встроенное объемное соотношение 2-8 [27] и могут работать при высоком давлении около 8 МПа [28] и температуре около $150{ }^{\circ} \mathrm{C}$ [29]. Наибольшая изоэнтропическая эффективность и объемная эффективность для лопастного расширителя составляют 71 и 57 \% соответственно, в то время как мощность расширителя составляет от нескольких ватт до 2,2 кВт [30]. Низкая изоэнтропическая эффективность и мощность связаны с утечкой и потерями на трение расширителя [31]. Они имеют низкую скорость вращения, 1500-3000 об/мин, и могут быть непосредственно присоединены к генератору.

Винтовой расширитель состоит из винтовых роторов с зазором около 50 мкм и бывает как одновинтовой, так и двухвинтовой. У них простая структура, средняя фракционность и утечки, средний шум и относительно высокая стоимость. У винтовых расширителей соотношение давлений 2-10 и объемное соотношение 2-8 [32]. Мощность выходного винта расширителя варьируется от 1,5 кВт до 1 МВт [33], что указывает на их уникальные характеристики для широкого диапазона мощности и размеров по сравнению с остальными объемными расширителями. Представленная изоэнтропическая эффективность варьирует от 20 до 70 \% [33]. Винтовой расширитель обычно не рекомендуется для размера менее 10 кВт из-за потерь на утечки и точных требований к механической обработке [34], что приводит к высокой стоимости расширителя. Они могут работать при $190^{\circ} \mathrm{C}$ и 1,6 МПа [35] и в двухфазной зоне, поскольку жидкость служит в качестве наполнителя между зазорами ротора и уменьшает потери на утечку.

В настоящее время поршневые расширители используются в мини-ТЭЦ, для утилизации отработанного тепла из двигателей внутреннего сгорания и холодильных циклов [36]. Наибольшая изоэнтропическая эффективность составляет 76 \% [37], но в большинстве литературных источников сообщаемая эффективность расширителей ниже 50 \%. Поршневые расширители могут работать при высоком давлении и температуре -9 МПа и $380-560{ }^{\circ} \mathrm{C}$ [36]. Они имеют более низкую скорость, 600-2000 об/мин, чем лопастные и винтовые расширители. Более низкая скорость позволяет подключение расширителя к генератору минуя редуктор для уменьшения скорости. Поршневые расширители могут применяться для больших отношений давления, особенно для закритического цикла $\mathrm{CO}_{2}$, из-за более высокого внутреннего объемного соотношения, внутреннее объемное соотношение поршневых расширителей колеблется от 6 до 14 [38]. Большая часть поршневых расширителей, которые были ранее изучены, имели мощность ниже 2 кВт, за исключением исследования [36], в котором сообщалось о мощности 18,6 кВт с водой в качестве рабочей жидкости.

Спиральный расширитель представляет собой машину с принудительным вытеснением и имеет фиксированное встроенное соотношение объема. Он состоит из двух спиралей, вращающейся спирали и фиксированной спирали. Спиральные расширители имеют встроенное соотношение объема 1,5-5 [39], а максимальная мощность расширителя составляет 12 кВт, максимальная величина изоэнтропической эффективности - 83 \% [40]. Они могут выдерживать

$$
-739-
$$


рабочее давление 8,2 МПа и температуру $180{ }^{\circ} \mathrm{C}[40,41]$. Стандартное объемные соотношения составляет от 1,5 до 4, для более высоких объемных соотношений расширения расширители должны соединяться последовательно [41]. Соотношение давлений спиральных компрессоров обычно меньше 10, но в режиме расширителя соотношение давления достигает 15 [42]. Двигающаяся спираль нуждается в смазке, так как она не ограничена и вызывает значительный износ, если не смазывается. В движущейся спирали можно уменьшить потери на утечку при надлежащей смазке. Смазка спирали делает ОЦР более сложным. Необходим внешний контур смазки. Для удлиненных спиральных расширителей используется наконечник с низким коэффициентом трения, уменьшающий радиальные утечки. При дальнейшей масляной смазке потери утечки могут быть дополнительно уменьшены. Смазочное масло служит зазором. Производительность расширителя зависит от геометрии, а также от рабочей жидкости и рабочих условий.

Авторами [43] был произведен анализ объемных расширителей по техническим, экономическим и коммерческим показателям. В результате винтовой и спиральный расширители оказались наиболее эффективными. Тем не менее винтовые расширители не подходят для производства электроэнергии менее 25 кВт из-за своей высокой стоимости. Поэтому спиральные расширители являются наиболее подходящими объёмными расширителями в диапазоне 1-25 кВт из-за простой конструкции, способности работать с двухфазными телами, низкой стоимости и относительно высокой изоэнтропийной эффективности. Хотя поршневые расширители обеспечивают большую степень расширения и высокую изоэнтропийную эффективность, они имеют сложную конструкцию и не могут работать при двухфазном рабочем теле, подвергаются вибрации во время работы и требуют частой балансировки.

\section{Особенности проектирования установок ОЦР}

Установка, работающая на органическом цикле Ренкина, должна обеспечивать надежное производство электрической энергии, контролировать основные параметры, иметь запас рабочего тела на пуск и останов установки. Схема установки, соответствующая данным требованиям, представлена на рис. 3. Заполнение рабочим теплом установки производится в ресивер, также он служит запасом рабочего тела при останове. Сепаратор добавляется в установку для удаления из жидкой фазы паров фреона после исправителя и увеличения срока службы расширителя. Цикл испаритель - сепаратор - ресивер - питательный насос является пусковым, как только температура фреона становится равной расчетной, он поступает на расширитель. Для контроля параметров и переключения между режимами установки была разработана схема КИПиА (рис. 4).

\section{Проектирование установки ОЦР мощностью 4 кВТ}

Проектирование установки начинается с определения источника тепла и его температуры. В данной установке источником тепла является горячая вода от котла Прометей-40 Автомат. Моделирование и расчет параметров схемы выполнялись с помощью калькулятора CoolProop и программы SmoWeb. На рис. 5 представлена диаграмма цикла данной установки, в табл. 3 основные параметры установки, в табл. 4 - основные параметры по точкам. Выбор рабочего тела для данной температуры источника тепла производился с учетом термодинамической эф-

$$
-740-
$$




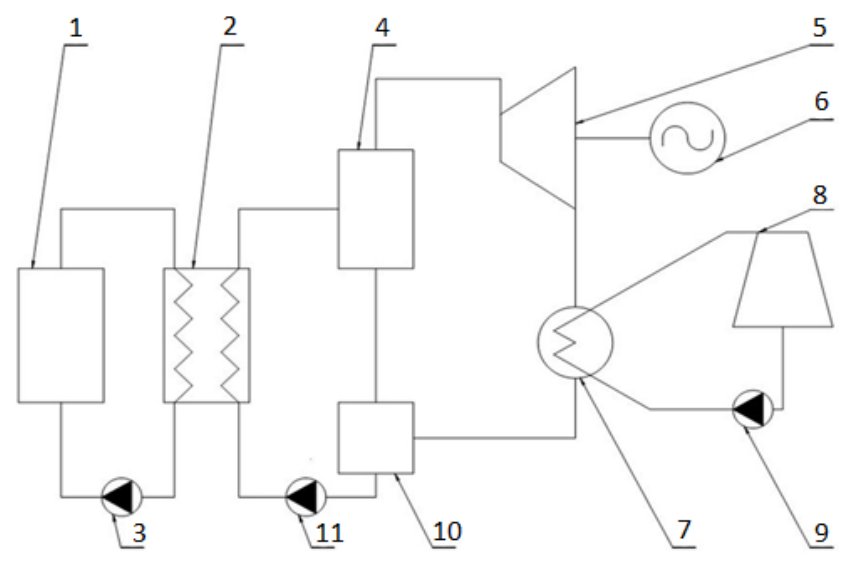

Рис. 3. Технологическая схема: 1 - источник тепловой энергии; 2 - испаритель; 3 - подающий насос; 4 сепаратор; 5 - турбина; 6 - электрогенератор; 7 - конденсатор; 8 - градирня; 9 - циркуляционный насос; 10 - ресивер; 11 - питательный насос

Fig. 3. Technological scheme: 1 - source of thermal energy; 2 - evaporator; 3 - feed pump; 4 - separator; 5 turbine; 6 - electric generator; 7 - capacitor; 8 - cooling tower; 9 - circulation pump; 10 - receiver; 11 - feed pump

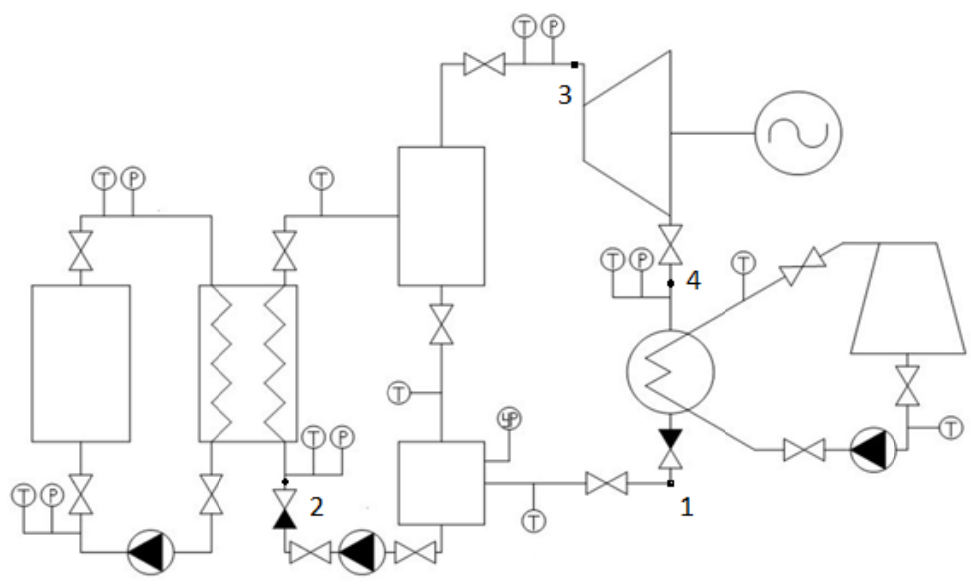

Рис. 4. Схема КИПиА

Fig. 4. Instrumentation diagram

фективности (теплоперепада на расширителе), экологических показателей и наличия в Красноярске. Выбранным рабочим телом является фреон R142b.

По расчетным данным было подобрано оборудование и смоделирована установка. На рис. 6 представлена 3D-модель установки.

\section{Заключение}

Увеличение интереса и реализация утилизации низкопотенциальной энергии способствуют развитию нетрадиционной энергетики и, как следствие, одной из самых перспективных технологий для этого органического цикла Ренкина. На сегодняшний момент эта технология 


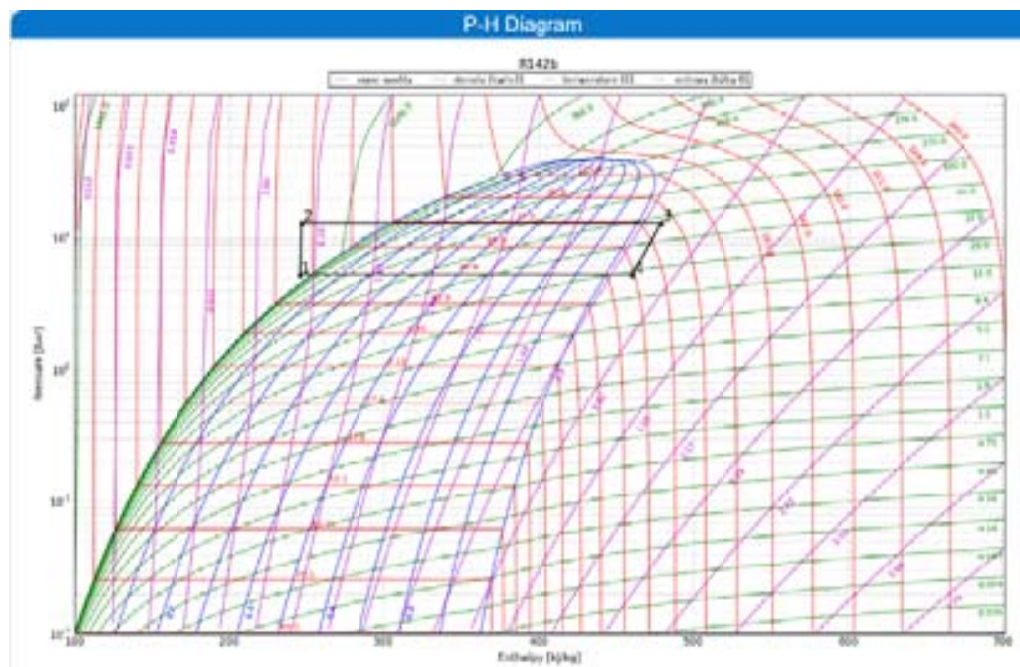

Рис. 5. Р-Н-диаграмма цикла установки

Fig. 5. The P-H diagram Installation Cycle

Таблица 3. Основные параметры установки

Table 3. basic installation parameters

\begin{tabular}{|l|c|}
\hline \multicolumn{1}{|c|}{ Параметр } & Значение \\
\hline Температура источника тепла, ${ }^{\circ} \mathrm{C}$ & 95 \\
\hline Мощность установки, кВт & 4 \\
\hline КПД установки, \% & 10 \\
\hline Габариты, мм & $2000 \times 800 \times 1500$ \\
\hline Масса & 150 кг \\
\hline
\end{tabular}

Таблица 4. Параметры в точках

Table 4. Parameters in points

\begin{tabular}{|l|c|c|c|c|}
\hline \multicolumn{1}{|c|}{ Точка } & 1 & 2 & 3 & 4 \\
\hline Давление, МПа & 0,523 & 1,3 & 1,3 & 0,523 \\
\hline Температура, ${ }^{\circ} \mathrm{C}$ & 35 & 35,4 & 90 & 57,21 \\
\hline Энтальпия, кДж/кг & 245,43 & 246,15 & 479,47 & 460,65 \\
\hline Плотность, кг/ & 1085,62 & 1087,75 & 479,47 & 460,65 \\
\hline
\end{tabular}

является зрелой, но не до конца исследованной; особый интерес для ученых и исследователей представляет выбор рабочего тела и расширителя. В этой статье изложен известный опыт по данным вопросам и подход к моделированию таких установок. 

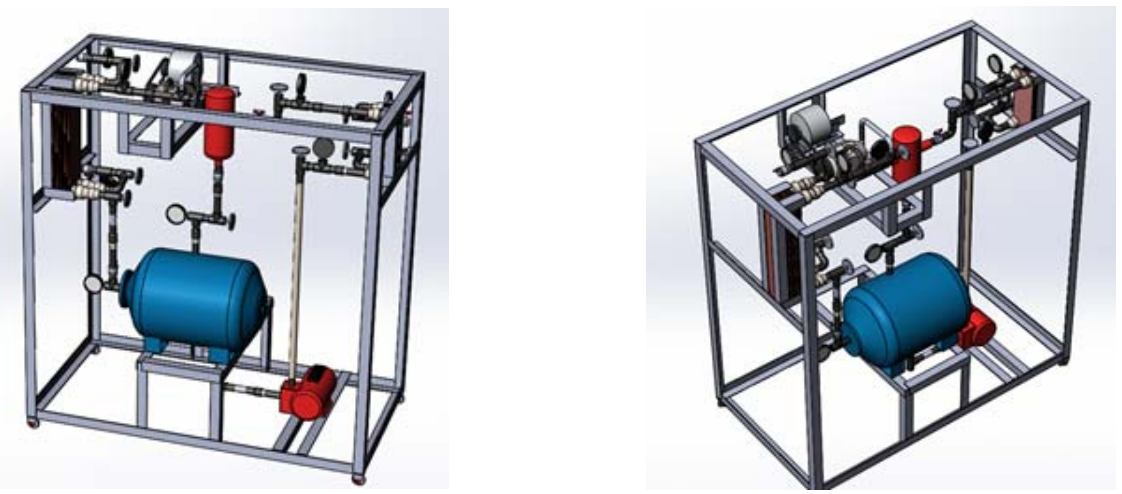

Рис. 6. 3D-модель установки

Fig. 6. 3D installation model

\section{Список литературы}

[1] M. Bianchi., A. De Pascale. Bottoming cycles for electric energy generation: Parametric investigation of available and innovative solutions for the exploitation of low and medium temperature heat sources, Applied Energy, 2011, 88, 1500-1509.

[2] Карабарин Д.И. Анализ методов утилизации теплоты анодных газов в алюминиевом производстве. Промышиленная энергетика, 2017, 1, 25-29 [Karabarin D.I. Analysis of methods of utilization of heat of anode gases in aluminum production, Industrial energy, 2017, 1, 25-29 (In Russian)]

[3] Карабарин Д.И., Михайленко С.А. Проект автономной котельной в районах децентрализованной энергетики “НАУКА ТЕХНОЛОГИИ ИННОВАЦИИ» часть 4. НГТУ, 2017, 1, 62-66 [Karabarin D.I., Mihailenko S.A. Project of an Autonomous boiler house in the areas of decentralized energy SCIENCE TECHNOLOGY INNOVATION" part 4. NGTU, 2017, 1, $62-66$ (In Russian)]

[4] Quoilin S., Van Den Broek M., Declaye S., Dewallef P., Lemort V.. Techno economic survey of Organic Rankine Cycle (ORC) systems. Renewable and Sustainable Energy Reviews, 2013, 22, 168-186.

[5] Vanslambrouck B. The Organic Rankine Cycle: current market overview. In: Proceedings of the international symposium on waste heat recovery by Organic Rankine Cycle, 2009.

[6] Ohman H. Implementation and evaluation of a low temperature waste heat recovery power cycle using NH3 in an Organic Rankine, Cycle Energy, 2012, 2, 74-80.

[7] Citrin D. Power generation from cement plant waste heat (power point presentation). CIIGreen Cementech: ORMAT InternationalInc, 2005.

[8] Gaia M. Turboden ORC Systems "Electricity Generation from Enhanced Geothermal Systems", Strasbourg, 2006.

[9]LorenzM.Biomasse-KWK mit einem Dampf-Schraubenmotor, Kohler\&Ziegler Anlagentechnik GmbH, 2006.

[10] Holdmann G. ORC technology for waste heat applications. In: Proceedings of the diesel heat recovery and efficiency workshop, 2007.

[11] Schuster A, Karellas S, Kakaras E, Spliethoff H. Energetic and economic investigation of Organic Rankine Cycle applications, Applied Thermal Engineering, 2008, 29, 1809-1817. 
[12] Quoilin S., Lemort V. Technological and economical survey of Organic Rankine Cycle systems. In: Proceedings of the ECEMEI conference, Vilamoura, 2009.

[13] Zyhowski G., Brown A. Low Global Warming Fluids for Replacement of HFC-245fa and HFC-134a in ORC Applications Honeywell - A, History of Innovation CFCs HCFCs HFCs HFOs, 2014.

[14] Chen Q., Xu J., Chen H., A new design method for Organic Rankine Cycles with constraint of inlet and outlet heat carrier fluid temperatures coupling with the heat source, Apply Energy, 2012, $98,562-573$.

[15] Guo C., Du X., Yang L., Yang Y., Organic Rankine cycle for power recovery of exhaust flue gas, Applied Thermal Engineering, 2015, 75, 135-144.

[16] Yang K., Zhang H., Wang Z., Zhang J., Yang F., Wang E., Yao B. Study of zeotropic mixtures of ORC (organic Rankine cycle) under engine various operating conditions, Energy, 2013, 58, 494-510.

[17] Garg P., Kumar P., Srinivasan K., Dutta P., Evaluation of isopentane, R-245fa and their mixtures as working fluids for organic Rankine cycles, Applied Thermal Engineering, 2013, 51, 292 300.

[18] Wang X. D., Zhao L. Analysis of zeotropic mixtures used in low-temperature solar Rankine cycles for power generation Solar Energy, 2009, 83(5), 605-613.

[19] Tchanche B.F., Lambrinos G., Frangoudakis A., Papadakis G. Low-grade heat conversion into power using organic Rankine cycles - A review of various applications, Renewable and Sustainable Energy Reviews, 2011, 15(8), 3963-3979.

[20] Xu J., Yu C. Critical temperature criterion for selection of working fluids for subcritical pressure Organic Rankine cycle, Energy, 2014, 74, 719-733.

[21] Quoilin S. Sustainable energy conversion through the use of organic Rankine cycles for waste heat recovery and solar applications [Ph.D thesis]. University of Liège; 2011.

[22] Person J.G. Performance mapping vs design parameters for screw compressors and other displacement compressor types. VDI Berichte $n r .859$, Düsseldorf; 1990.

[23] Qiu G, Shao Y, Li J, Liu H, Riffat SB. Experimental investigation of a biomass- fired ORCbased micro-CHP for domestic applications, Fuel, 2012, 96, 374-382.

[24] Desideri A, Broek MVD, Gusev S, Lecompte S, Lemort V. Experimental study and dynamic modeling of a WHR ORC power system with screw expander. In: Proceedings of the ASME ORC 2013 2nd international seminar ORC power system, Rotterdam, Netherlands, 2013.

[25] Zheng N., Zhao L., Wang X.D., Tan Y.T. Experimental verification of a rolling- piston expander that applied for low -temperature Organic Rankine Cycle, Applly Energy, 2013, 112, 1265-1274.

[26] Lemort V., Declaye S., Quoilin S. Experimental characterization of a hermetic scroll expander for use in a micro-scale Rankine cycle, Power Energy, 2011, 226, 126-136.

[27] Farrokhi M., Noie S.H., Akbarzadeh Aa. Preliminary experimental investigation of a natural gas-fired ORC-based micro-CHP system for residential buildings, Applly Thermal Enineering, 2014, 69(1), 221-229.

[28] Yang B., Peng X., He Z., Guo B., Xing Z. Experiment al investigation on the internal working process of a CO2 rotary vane expander buildings, Applly Thermal Enineering, 2009, 29, 2289-2296.

[29] Badr O., Probert S.D., O'Callaghan P. Performances of multi-vane expanders, Apply Energy, 1985, 20, 207-234. 
[30] Subiantoro A., Yap K.S., Ooi K.T. Experimental investigations of the revolving vane (RV-I) expander, Applly Thermal Enineering, 2013, 50, 393-400.

[31] Xia C., Zhang W., Bu G., Wang Z., Shu P. Experimental study on a sliding vane expander in the HFC410 A refrigeration system for energy recovery, Applly Thermal Enineering, 2013, 59, 559567.

[32] Brümmer P.A. Energy efficiency - waste heat utilization with screw expanders, Pumps, Compressors and Process Components, 2012

[33] Wang W.,Wu Y., Ma C., Xia G., Wang J. Experimental study on the performance of single screw expanders by gap adjustment, Energy, 2013, 62, 379-384.

[34] Qiu G., Liu H., Riffat S. Expanders for micro-CHP systems with organic rankine cycle Applly, Thermal Enineering, 2011, 31, 3301-3307.

[35] Zhang Y. Experimental study on the performance of single screw expander with $195 \mathrm{~mm}$ diameter screw. In: Proceedings of the ASME ORC 2013 2nd International Seminar ORC Power System Rotterdam, Netherlands; 2013.

[36] Seher D., Lengenfelder T., Gerhardt J., Eisenmenger N., Hackner M., Krinn I. Waste heat recovery for commercial vehicles with a rankine process. In: 21st Aachen colloquium automobile and engine technology, Aachen, Germany; 2012.

[37] Wang X.D., Zhao L.,Wang J.L., Zhang W.Z., Zhao X.Z., Wu W. Performance evaluation of a low-temperature solar Rankine cycle system utilizing R245fa, Solar Energy, 2010, 84, 353-364.

[38] Lemort V., Guillaume L., Legros A., Declaye S. A comparison of piston, screw and scroll expanders for small-scale Rankine cycle systems. 3rd Int. Conf. Microgeneration Relat. Technol. Naples, Italy; 2013.

[39] Clemente S., Micheli D., Reini M., Taccani R. Energy efficiency analysis of organic rankine cycles with scroll expanders for cogenerative applications, Apply Energy, 2012, 97, 792-801.

[40] Zhou N., Wang X., Chen Z., Wang Z. Experimental study on organic rankine cycle for waste heat recovery from low-temperature flue gas, Energy, 2013, 55, 216-225.

[41] Oudkerk J.F., Quoilin S., Lemort V. Evaluation of an orc based micro-CHP system involving a hermetic scroll expander.In: Proceedings of the ASMEORC 2011, first international seminar on ORC power systems. TU delft, Netherlands; 2011.

[42] Clemente S., Micheli D., Reini M. Numerical model and performance analysis of a scroll machine for ORC applications. In: Proceeding of ECOS 2010, Lausanne, Switzerland; 2010.

[43] Muhammad I., Muhammad U., Byung S.P., Dong H.L. Volumetric expanders for low grade heat and waste heat recovery applications, Renewable and Sustainable Energy Reviews, 2016, 57, 1090-1109. 